\title{
Threshold Characteristics of Slow-Light Photonic Crystal Lasers
}

Xue, Weiqi; Yu, Yi; Ottaviano, Luisa; Chen, Yaohui; Semenova, Elizaveta; Yvind, Kresten; Mørk, Jesper

Published in:

Physical Review Letters

Link to article, DOI:

10.1103/PhysRevLett.116.063901

Publication date:

2016

Document Version

Publisher's PDF, also known as Version of record

Link back to DTU Orbit

Citation (APA):

Xue, W., Yu, Y., Ottaviano, L., Chen, Y., Semenova, E., Yvind, K., \& Mørk, J. (2016). Threshold Characteristics of Slow-Light Photonic Crystal Lasers. Physical Review Letters, 116(6), [063901].

https://doi.org/10.1103/PhysRevLett.116.063901

\section{General rights}

Copyright and moral rights for the publications made accessible in the public portal are retained by the authors and/or other copyright owners and it is a condition of accessing publications that users recognise and abide by the legal requirements associated with these rights.

- Users may download and print one copy of any publication from the public portal for the purpose of private study or research.

- You may not further distribute the material or use it for any profit-making activity or commercial gain

- You may freely distribute the URL identifying the publication in the public portal 


\title{
Threshold Characteristics of Slow-Light Photonic Crystal Lasers
}

\author{
Weiqi Xue, ${ }^{*}$ Yi Yu, Luisa Ottaviano, Yaohui Chen, Elizaveta Semenova, Kresten Yvind, and Jesper Mork \\ DTU Fotonik, Department of Photonics Engineering, Technical University of Denmark, Building 343, \\ DK-2800 Kongens Lyngby, Denmark
}

(Received 31 July 2015; published 9 February 2016)

\begin{abstract}
The threshold properties of photonic crystal quantum dot lasers operating in the slow-light regime are investigated experimentally and theoretically. Measurements show that, in contrast to conventional lasers, the threshold gain attains a minimum value for a specific cavity length. The experimental results are explained by an analytical theory for the laser threshold that takes into account the effects of slow light and random disorder due to unavoidable fabrication imperfections. Longer lasers are found to operate deeper into the slow-light region, leading to a trade-off between slow-light induced reduction of the mirror loss and slow-light enhancement of disorder-induced losses.
\end{abstract}

DOI: 10.1103/PhysRevLett.116.063901

Slow light in photonic crystal (PC) line-defect waveguides [1] enhances the interaction between the propagating light wave and the material of the waveguide, and has enabled the demonstration of increased material nonlinearity [2], enhanced spontaneous emission into the propagating mode [3,4], and enhanced material gain [5]. Microcavity lasers can be realized in the same PC membrane structure by exploiting high-quality point-defect cavities and in the past decade significant progress was made [6-9], culminating in recent demonstrations of highspeed electrically pumped structures [10]. Such PC lasers allow the exploration of new operation regimes, such as single emitter lasing [11] and ultrahigh speed modulation [12]. However, while it was shown that slow light in combination with random spatial disorder leads to very rich physics [13-19], the role of slow light on lasers realized using defect cavities has apparently not been systematically investigated. For the case of passive point-defect cavities, it is well known that disorder is an important factor limiting the quality factor [20-23] but the role of slow light in extended active cavities is not well understood.

In this Letter, we report experimental results on PC quantum dot lasers with variable cavity length and show that these attain a minimum threshold gain for a certain cavity length, in stark contrast to conventional lasers, where the threshold gain decreases monotonically with cavity length. These results show that disorder may lead to fundamental limitations on the performance of nanostructured lasers, but the results also demonstrate a promising platform for investigating disorder effects in active structures.

The PC cavity lasers investigated here are realized in a $250 \mathrm{~nm}$ thick InP air-embedded PC membrane [24] with three layers of quantum dots (QDs) with densities of $\sim 5.4 \times 10^{10} \mathrm{~cm}^{-2}$, extending over the entire membrane. QDs are preferred over quantum wells for these studies due to a low surface recombination and better thermal characteristics. Details about the fabrication and optical properties of the QDs can be found in [25]. The PC structure has a lattice constant of $a=438 \mathrm{~nm}$ and an air-hole radius of $0.25 a$. A so-called $L N$ cavity [26] is formed by omitting $N$ air holes in a $W 1$ defect waveguide. Structures with different cavity lengths, i.e., $L 1-L 20$, are fabricated and characterized. In Fig. 1(a), the red solid curve illustrates the dispersion of the even TE-like $W 1$-waveguide mode. As the cavity length increases, the position of the fundamental mode moves closer to the Brillouin zone (BZ) edge, as shown by markers, where the group index strongly increases [26], thus allowing a systematic investigation of the role of slow light on the laser properties.

The samples are pumped and monitored vertically using a microphotoluminescence setup [24]. All measurements are performed at room temperature. Figure 1(b) shows a typical example of a measured light-out vs light-in
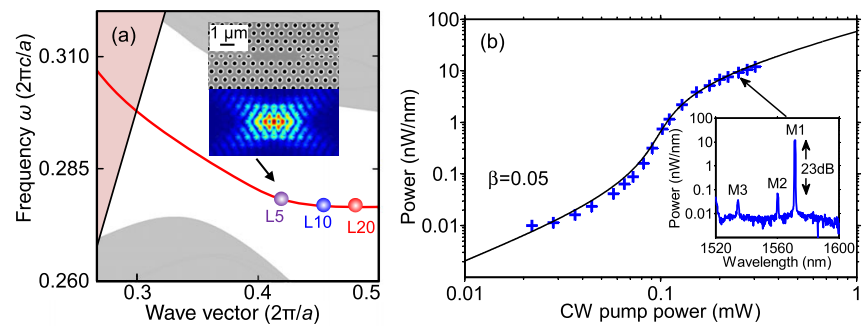

FIG. 1. (a) Illustration of the PC band diagram for the TE-like even waveguide mode (solid line) and positions of the fundamental cavity mode for L5, L10, and L20 defect cavities (markers). Shaded areas indicate the light cone and nonguided modes of the structure. The inset shows a SEM image of an L5 PC cavity laser and the simulated spatial mode profile of the electric field amplitude. (b) Measured power of the fundamental mode $M 1$ vs pump power and corresponding theoretical fit (solid line) for an $L 9$ laser. The inset shows the output spectrum far above threshold. 
characteristic, obtained by pumping an $L 9$ cavity with a cw $1480 \mathrm{~nm}$ beam. The pump power is estimated by multiplying the excitation power $\left(P_{\text {exc }}\right)$ with the ratio $\left(\Gamma_{\text {exc }}\right)$ of the PC cavity area to the total pump area. The inset of Fig. 1(b) shows the dominance of the fundamental lasing mode, denoted $M 1$, but also the presence of two higher order longitudinal cavity modes, $M 2$ and $M 3$. The solid line in Fig. 1(b) is a fit to a conventional laser rate equation model, see, e.g., [10], leading to a laser threshold of $\sim 90 \mu \mathrm{W}$ and a spontaneous emission factor of $\beta=0.05$. This $\beta$ factor is somewhat smaller than values reported in [10], primarily because of a wide gain spectrum and the lack of transverse confinement of the gain medium in our structures. The relatively small $\beta$ factor enables a precise extraction of the threshold, and also ensures that we are in a large-cavity limit, where the total spontaneous emission rate does not depend appreciably on cavity length.

Figure 2 summarizes the measured variation of the laser threshold with the cavity length for three sample bars with the same PC design. The threshold pump density is defined as $P_{\mathrm{exc}} \Gamma_{\mathrm{exc}} / L$, where $L$ is the cavity length. While bars 1 and 2 were located close to each other on the wafer and fabricated in the same run, bar 3 is more distant and is from another processing batch. Gain material variations and fabrication uncertainties are believed to account for the higher threshold of sample bar 3. The error bar indicates the largest variation of measured threshold between two repeated measurements. Lasing was not observed for $L 1$, $L 2$, and $L 3$ cavities, which is attributed to the increase of the mirror losses, requiring strong pumping and leading to additional heating. Despite the difference in absolute gain level, all of the sample bars show the same qualitative behavior. From $L 4$ to $L 8 / L 9$, the threshold decreases monotonically with cavity length after which it increases. An optimum cavity length thus exists, for which the laser threshold density attains a minimum. This behavior is strikingly different from the case of conventional edge emitting lasers, where the threshold density decreases monotonically with length since the mirror losses decrease in inverse proportion to the cavity length.

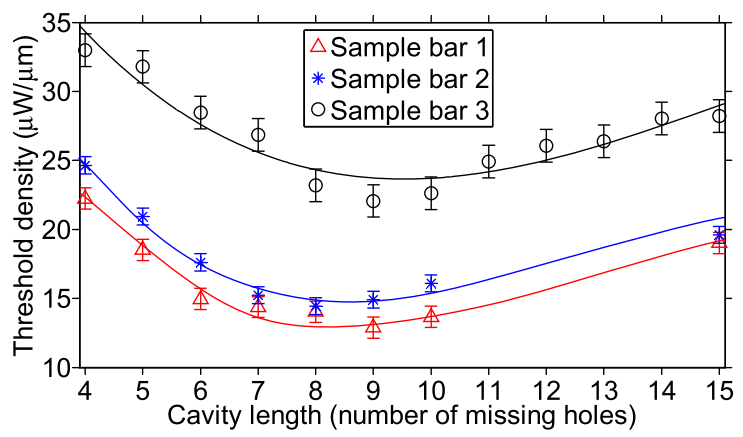

FIG. 2. Measured threshold pump power density versus cavity length. The solid lines are guides to the eye.
In the measurements reported in Fig. 2, we have taken a number of precautions to eliminate other causes for threshold variation. In particular, the pump profile is kept fixed with a diameter of $20 \mu \mathrm{m}(\sim 46 a)$, which is much larger than the extent of all of the investigated cavities from $L 1$ to L15. This ensures a uniform illumination, avoiding a systematic reduction of the pump efficiency with cavity length. Heating induced by the optical pump beam leads to a redshift of the laser wavelength. The use of an InP membrane already significantly improves the thermal properties compared to more conventional InGaAsP structures [24]. To further reduce the thermal effect, we employed pulsed pumping with a pulse width of $500 \mathrm{~ns}$ and a low duty cycle of $2 \%$. The spectral variation of the lasing mode might also affect the threshold gain via a wavelength dependent material gain. However, due to the inhomogeneous size distribution of our QDs, the gain spectrum is very wide (the photoluminescence spectral width is larger than $150 \mathrm{~nm}$ ) and furthermore the mode frequency is almost independent of cavity length beyond L8 cavities, cf. Fig. 3(a). Therefore, the variation of material gain is insignificant.

The variation of the experimentally observed cavity mode frequencies with cavity length is shown by the "square" markers in Fig. 3(a). As the cavity length increases, all modes shift to lower frequencies and the spacing between neighboring modes decreases. Qualitatively similar behavior was observed in [8]. From the mode spacing and the known cavity length we can calculate the effective group refractive index. The extracted

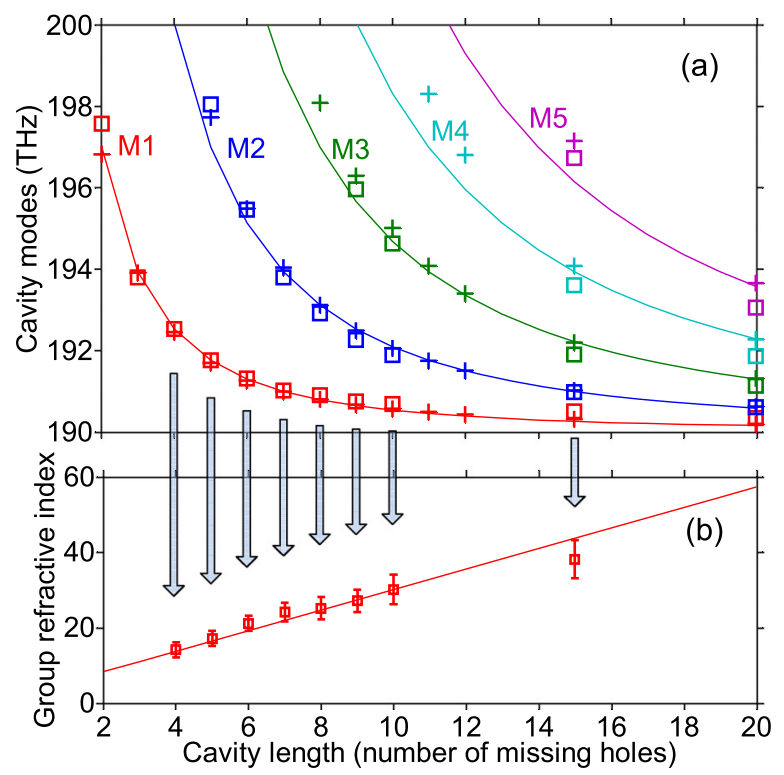

FIG. 3. (a) Measured cavity mode frequencies ("square" markers) versus cavity length. The "cross" markers are full numerical calculations. (b) The measured group refractive index (markers) for the fundamental mode $M 1$. Solid lines are the predictions of Eq. (2). 
group index for the fundamental $M 1$ mode, Fig. 3(b), increases monotonically with the cavity length, consistent with the mode approaching the BZ edge. For the optimum cavity length, the group index is of the order of $\sim 20$.

It is already known that disorder in PC waveguides leads to propagation losses that strongly increase in the slowlight region $[13,18]$. Since the fundamental mode moves deeper into the slow-light region upon increasing the cavity length, it is perhaps not surprising that an optimum cavity length exists. However, since the effective material gain per unit length also increases in the slow-light region [5], and the positive role of slow light on promoting laser oscillation in similar-type lasers was suggested [27], a detailed analysis is required.

We model the laser cavity as an effective Fabry-Perot resonator, but with the propagating wave to be a Bloch mode of the $W 1$ waveguide. This was shown in [28] to be a good approximation even for defect cavities as short as a few lattice periods. The effective length of the cavity is $L \cong(N+1) a$, where $N$ is the number of missing holes. By requiring that the Bloch mode experiences a round-trip phase change of an integer times $2 \pi[29,30]$, we find that the $M$ th cavity mode is displaced by $\triangle k \cong M \pi / L$ from the BZ edge located at $k_{0}=\pi / a$ [26]. Close to this band minimum we may expand the dispersion to second order:

$$
\omega(k) \cong \omega\left(\frac{\pi}{a}\right)+\frac{1}{2} \frac{d^{2} \omega}{d k^{2}}(\triangle k)^{2} .
$$

The deviation of the mode frequency from the BZ edge, $\triangle f$, and the slow-down factor, $S=n_{g}^{\mathrm{PC}} / n_{g}^{b}=v_{g}^{b} / v_{g}^{\mathrm{PC}}$, thus become

$$
\triangle f=\frac{v_{g}^{b} l_{2}}{4} \frac{M^{2}}{L^{2}} ; \quad S=\frac{L}{M l_{2}} .
$$

Here, $v_{g}^{x}\left(n_{g}^{x}\right)$ is the group velocity (group refractive index) in the PC cavity $(x=\mathrm{PC})$ and in the corresponding homogeneous material $(x=b)$, and $l_{2}=\left(\pi / v_{g}^{b}\right)\left(d^{2} \omega /\right.$ $\left.d k^{2}\right)$ is a characteristic length scale, inversely proportional to an effective photon mass.

The predictions of Eq. (2) are shown by the solid lines in Fig. 3, using $n_{g}^{b}=3.17, l_{2}=510 \mathrm{~nm}$, and a band-edge frequency of $190 \mathrm{THz}$. The good agreement with experiment supports the validity of our approximate analytical model. Mode frequencies ["cross" markers in Fig. 3(a)] obtained from 3D finite-element simulations without adjustable dispersion parameters further confirm the model.

A rate equation for the cavity photon density $N_{p}$ is derived by considering the incremental change during one cavity round-trip time $\tau_{R}=2 S L / v_{g}^{b}$. Here, we focus on the fundamental mode $M 1$ and the laser can be approximately considered as a single-mode laser because of the dominance of $M 1$. The modal gain per unit length of a Bloch wave propagating in the structure is $g_{\text {mod }}=\Gamma S g_{\text {mat }}$, where $g_{\text {mat }}$ is the material gain coefficient for the homogeneous active medium and $\Gamma$ is the confinement factor [5]. Neglecting spontaneous emission

$$
\frac{d N_{p}}{d t}=v_{g}^{b}\left[\Gamma g_{\mathrm{mat}}-\left(\alpha_{\mathrm{WG}}+\alpha_{\mathrm{MIR}}\right) / S\right] N_{p} .
$$

Here, $\quad \alpha_{\mathrm{MIR}}=\ln \left[1 /\left(R_{1} R_{2}\right)\right] /(2 L)$ and $\alpha_{\mathrm{WG}}$ denote the mirror and waveguide loss. We see that the spatial gain enhancement due to slow light is canceled out by the longer round-trip time, which is basically a statement that the rate of gain per unit time is unaffected by slow light $[5,27]$. Fabrication induced disorder approximately scales as $\alpha_{\mathrm{WG}}=\alpha_{1} S+\alpha_{2} S^{2}[13,18]$, where the first term accounts for out-of-plane scattering and absorption losses and the second term represents backscattering. Since the latter component does not add coherently to the laser field we assume that it too acts as a loss term. From this rate equation, the threshold gain becomes

$$
\Gamma g_{\mathrm{th}}=\alpha_{1}+\alpha_{2} S+\frac{1}{2 S L} \ln \left(\frac{1}{R_{1} R_{2}}\right) \text {. }
$$

We see that the mirror loss is reduced in inverse proportion to the slow-down factor $S$, while disorder-induced losses originating from backscatter increase linearly with the slow-down factor. Since $S$ itself scales linearly with $L$, short lasers are dominated by mirror loss, while long lasers are governed by disorder-induced losses. Figure 4 shows the calculated length dependence of the modal threshold gain, $\Gamma g_{\mathrm{th}}-\alpha_{1}$, for the fundamental mode. For $\alpha_{2}=0 \mathrm{~cm}^{-1}$, the threshold gain decreases monotonically with $L$, as for a conventional semiconductor laser, but for nonzero $\alpha_{2}$, an optimum cavity length is found, in good qualitative agreement with the experimental observations. For the calculations presented here, we used wavelength independent mirror reflectivities of $R_{1}=R_{2}=0.98$, but qualitatively similar results are obtained using a wavelength dependent reflectivity, as found, e.g., in [31]. For our structure, the standard deviation of the variation of hole radius was measured to be of the order of $1 \mathrm{~nm}$. A

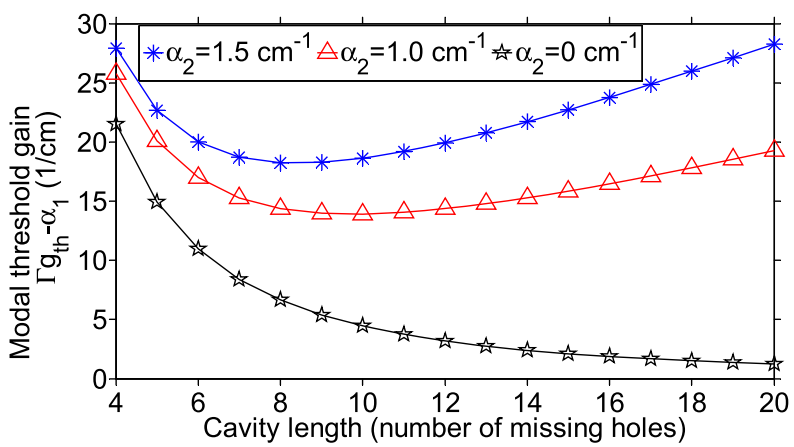

FIG. 4. Calculated threshold gain, $\Gamma g_{\text {th }}-\alpha_{1}$, vs cavity length for different values of $\alpha_{2} . l_{2}=510 \mathrm{~nm}, R_{1}=R_{2}=0.98$. 
quantitative prediction of the lasing threshold will require a knowledge of the detailed variation of the coefficients $\alpha_{1}$, $\alpha_{2}, R_{1}$, and $R_{2}$ with wavelength, spatial mode distribution, and fabrication procedure, which is the topic of future work.

From these results we infer that, in the presence of disorder, the quality factor of a PC cavity is maximized for a specific cavity length. In order to further corroborate our finding we have addressed the problem using a different theoretical approach. In [32] it was shown that the loss of disordered waveguides can be efficiently computed by solving a generalized eigenvalue problem formulated in a basis of Bloch modes of the regular structure. Disorder leads to scattering among the Bloch modes and induces extrinsic losses of the guided modes, since these acquire Bloch mode components within the light cone. We apply the approach of [32] to the case of a cavity, which is represented by a stepwise variation of the average permittivity along the propagation direction of the cavity, corresponding to an effective-index approach. Considering only a single band, the eigenvalue problem can be formulated as

$$
\mathbf{D} \mathbf{v}_{\beta}=\omega_{\beta}(\mathbf{I}+\mathbf{V}) \mathbf{v}_{\beta} .
$$

Here, $\mathbf{D}$ is a diagonal matrix in the basis of Bloch modes, indexed by $k$, with $\sqrt{D_{k k}}=\omega_{k}$ being the waveguide dispersion. Furthermore, $\mathbf{I}$ is the identity matrix and $\mathbf{V}=\mathbf{V}^{\text {cavity }}+\mathbf{V}^{\text {disorder }}$ is the scattering matrix coupling (hybridizing) the waveguide Bloch modes due to a determinsitic cavity "potential" as well as a random "disorder" potential. The solution of Eq. (5) gives the eigenfrequencies of the cavity modes and the corresponding decomposition on Bloch modes. Figure 5 shows the calculated mode spectra for an $L 20$ cavity with and without the inclusion of disorder. A similar graphical representation was used in [33]. Upon including disorder, further scattering to the light cone takes place, reflecting the additional spatial frequency components introduced by the spatial disorder.

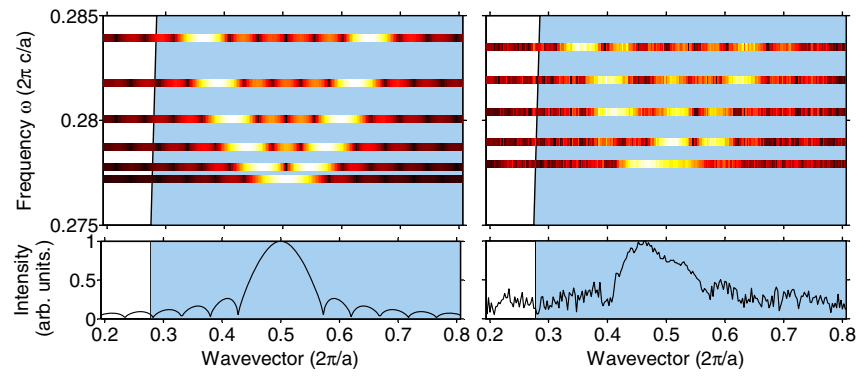

FIG. 5. Calculated cavity mode spectra for the L20 cavity without disorder (left column) and with disorder (right column). The Bloch mode distributions are shown in color scale for the calculated mode eigenfrequencies ( $y$ axis), with white (black) denoting high (low) intensity. The lower plots show the mode intensity for the fundamental mode versus wave vector. The white background area indicates the light cone.
Figure 6(a) shows the relative fraction of the mode spectrum within the light cone, which is an approximate measure of the loss rate, as a function of cavity length. An ensemble average over 500 realizations of the random scattering matrix $\mathbf{V}^{\text {disorder }}$ was performed. Quantitative results for the absolute loss rates can be obtained using the approach of [32], but it is beyond the scope of this paper. However, the features displayed by Fig. 6(a) are general: In the presence of disorder, the light cone intensity increases, lending support to the assumption of our analytical model that disorder-induced backscattering increases the cavity mode loss. Furthermore, the mode $M 1$ is found always to dominate. This is in accordance with our experimental findings, while the approximate analytical result Eq. (4) suggests that higher-order modes become dominant deep into the slow-light region.

We next comment on the applicability of these results to other types of line-defect cavities. Importantly, it has been demonstrated that the loss depends on the spatial mode distribution and can be engineered by modifying the rows of holes next to the line defect [18] and the $Q$ factor can be significantly increased by implementing a heterostructure cavity [34]. Figure 6(b) shows 3D finite-difference time domain simulations for these cavity types, demonstrating that in these cases the fundamental resonant mode also obeys a resonance condition $\triangle k \cong \pi / L$, where $L \cong N a+$ $l_{p}$ is the effective cavity length, including a finite penetration length $l_{p}$ into the mirrors. For both the standard and the dispersion-engineered structure, $l_{p} \cong a$, while for the heterostructure, $l_{p} \cong 2.3 a$, due to the slower and smoother variation of the field at the mirrors [34]. Thus, in all of these cases, as the cavity length increases, the fundamental mode approaches the BZ edge, where symmetry dictates that the band structure becomes flat and the group index, and consequently the disorder-induced losses, strongly increase. Thus, while design modifications will change the detailed variation of laser threshold with cavity length, the appearance of a minimum is expected for all three PC structures.

In conclusion, we have experimentally shown that PC $L$ cavity lasers display intriguing threshold characteristics,
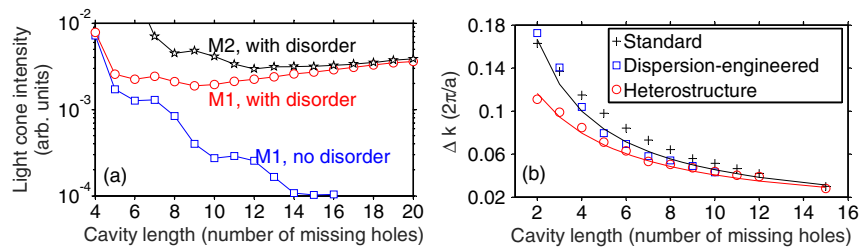

FIG. 6. (a) Calculated intensity within light cone vs cavity length for the fundamental mode $M 1$, with and without disorder, and for the higher-order mode $M 2$, with disorder. (b) The wave vector displacement of the fundamental mode from the BZ edge as a function of cavity length. Markers denote 3D finitedifference time domain simulations, while solid lines are obtained using $\triangle k \cong \pi / L$. 
with a minimum threshold gain being attained at a specific cavity length. An analytical model was derived that well accounts for the experimental results, suggesting that the observed threshold behavior is a result of the interplay between slow-light and disorder-induced losses. These results are important for the understanding and further development of photonic crystal lasers as well as demonstrating a platform for systematic investigations of disorder effects in active (amplifying) media and in cavities.

Financial support from Villum Fonden via the NATEC Center and the Young Investigator Programme Project QUEENs, and from the Danish Council for Independent Research (FTP 0602-02623b) are gratefully acknowledged. J. M. acknowledges helpful discussions with Professor S. Hughes, Queen's University, Canada.

*wexu@ fotonik.dtu.dk

[1] T. Baba, Nat. Photonics 2, 465 (2008).

[2] B. Corcoran, C. Monat, C. Grillet, D. J. Moss, B. J. Eggleton, T. P. White, L. OFaolain, and T. F. Krauss, Nat. Photonics 3, 206 (2009).

[3] S. Noda, M. Fujita, and T. Asano, Nat. Photonics 1, 449 (2007).

[4] T. Lund-Hansen, S. Stobbe, B. Julsgaard, H. Thyrrestrup, T. Sünner, M. Kamp, A. Forchel, and P. Lodahl, Phys. Rev. Lett. 101, 113903 (2008).

[5] S. Ek, P. Lunnemann, Y. Chen, E. Semenova, K. Yvind, and J. Mork, Nat. Commun. 5, 5039 (2014).

[6] H.-G. Park, S.-H. Kim, S.-H. Kwon, Y.-G. Ju, J.-K. Yang, J.-H. Baek, S.-B. Kim, and Y.-H. Lee, Science 305, 1444 (2004).

[7] S. Noda, Science 314, 260 (2006).

[8] K. A. Atlasov, M. Calic, K. F. Karlsson, P. Gallo, A. Rudra, B. Dwir, and E. Kapon, Opt. Express 17, 18178 (2009).

[9] S. Matsuo, A. Shinya, T. Kakitsuka, K. Nozaki, T. Segawa, T. Sato, Y. Kawaguchi, and M. Notomi, Nat. Photonics 4, 648 (2010).

[10] S. Matsuo, T. Sato, K. Takeda, A. Shinya, K. Nozaki, H. Taniyama, M. Notomi, K. Hasebe, and T. Kakitsuka, IEEE J. Sel. Topics Quantum Electron. 19, 4900311 (2013).

[11] M. Nomura, N. Kumagai, S. Iwamoto, Y. Ota, and Y. Arakaw, Nat. Phys. 6, 279 (2010).

[12] J. Mork, Y. Chen, and M. Heuck, Phys. Rev. Lett. 113, 163901 (2014).

[13] S. Hughes, L. Ramunno, J. F. Young, and J. E. Sipe, Phys. Rev. Lett. 94, 033903 (2005).
[14] S. Mazoyer, J. P. Hugonin, and P. Lalanne, Phys. Rev. Lett. 103, 063903 (2009).

[15] M. Patterson, S. Hughes, S. Schulz, D. M. Beggs, T. P. White, L. O'Faolain, and T. F. Krauss, Phys. Rev. B 80, 195305 (2009).

[16] N. LeThomas, H. Zhang, J. Jágerská, V. Zabelin, R. Houdré, I. Sagnes, and A. Talneau, Phys. Rev. B 80, 125332 (2009).

[17] L. Sapienza, H. Thyrrestrup, S. Stobbe, P. D. Garcia, S. Smolka, and P. Lodahl, Science 327, 1352 (2010).

[18] L. O'Faolain, S. A. Schulz, D. M. Beggs, T. P. White, M. Spasenović, L. Kuipers, F. Morichetti, A. Melloni, S. Mazoyer, J. P. Hugonin, P. Lalanne, and T. F. Krauss, Opt. Express 18, 27627 (2010).

[19] J. Liu, P. D. Garcia, S. Ek, N. Gregersen, T. Suhr, M. Schubert, J. Mork, S. Stobbe, and P. Lodahl, Nat. Nanotechnol. 9, 285 (2014).

[20] T. Asano, B.-S. Song, and S. Noda, Opt. Express 14, 1996 (2006).

[21] L. Ramunno and S. Hughes, Phys. Rev. B 79, 161303 (2009).

[22] S. L. Portalupi, M. Galli, M. Belotti, L. C. Andreani, T. F. Krauss, and L. O'Faolain, Phys. Rev. B 84, 045423 (2011).

[23] M. Minkov, U. P. Dharanipathy, R. Houdré, and V. Savona, Opt. Express 21, 28233 (2013).

[24] W. Xue, L. Ottaviano, Y. Chen, E. Semenova, Y. Yu, A. Lupi, J. Mork, and K. Yvind, Opt. Express 23, 18277 (2015).

[25] E. Semenova, I. Kulkova, S. Kadkhodazadeh, D. Barettin, O. Kopylov, A. Cagliani, K. Almdal, M. Willatzen, and K. Yvind, Proc. SPIE 8996, 899606 (2014).

[26] M. Okano, T. Yamada, J. Sugisaka, N. Yamamoto, M. Itoh, T. Sugaya, K. Komori, and M. Mori, J. Opt. 12, 075101 (2010).

[27] K. Kiyota, T. Kise, and N. Yokouchi, Appl. Phys. Lett. 88, 201904 (2006).

[28] P. Lalanne, C. Sauvan, and J. Hugonin, Laser Photonics Rev. 2, 514 (2008).

[29] S.-H. Kim, G.-H. Kim, S.-K. Kim, H.-G. Park, Y.-H. Lee, and S.-B. Kim, J. Appl. Phys. 95, 411 (2004).

[30] C. Bonato, J. Hagemeier, D. Gerace, S. M. Thon, H. Kim, L. C. Andreani, P. M. Petroff, M. P. van Exter, and D. Bouwmeestera, Photonics Nanostruct. Fundam. Appl. 11, 37 (2013).

[31] C. Sauvan, P. Lalanne, and J. P. Hugonin, Phys. Rev. B 71, 165118 (2005)

[32] V. Savona, Phys. Rev. B 83, 085301 (2011).

[33] K. A. Atlasov, M. Felici, K. F. Karlsson, P. Gallo, A. Rudra, B. Dwir, and E. Kapon, Opt. Express 18, 117 (2010).

[34] B.-S. Song, S. Noda, T. Asano, and Y. Akahane, Nat. Mater. 4, 207 (2005). 